\title{
A resolução oral de tarefas de divisão por crianças ${ }^{1}$
}

\author{
Jane Correa \\ Universidade Federal do Rio de Janeiro
}

\begin{abstract}
Resumo
O presente estudo examina as estratégias de resolução oral de tarefas de divisão partitiva e por quotas por crianças de 6 a 9 anos, com diferentes níveis de escolaridade em aritmética. Foram utilizados quatro valores para o dividendo (4, 8, 12 e 24) e dois divisores diferentes (2 e 4). O desempenho das crianças variou em função da idade e escolaridade, dado que estes fatores se confundem. De maneira geral, o desempenho das crianças foi influenciado pelo tamanho do dividendo e do divisor. Os procedimentos de dupla contagem e uso de fatos multiplicativos foram mais utilizados para a solução das tarefas de divisão por quotas enquanto que procedimentos baseados no uso de adições repetidas e estratégias envolvendo partição de quantidades foram relativamente mais empregados nos problemas de divisão partitiva. A pequena porcentagem de procedimentos baseados na subtração repetida sugere que esta não pode ser tomada como modo de representação intuitiva da divisão.

Palavras-chave: divisão partitiva; divisão por quotas; aritmética
\end{abstract}

\begin{abstract}
Children's mental solution to division tasks. The present study examines mental computational solutions to elementary partitive and quotitive problems given by children aged 6-9 with different levels of arithmetical knowledge. There were four sizes of dividend (4, 8, 12 and 24) and two different divisors (2 and 4). Children's performance improved both with age and schooling since these variables are confounded. Their performance was overall affected by both the size of the dividend and the divisor. Procedures based on double counting and multiplicative facts were more used in quotitive tasks. On the other hand, procedures related to repeated addition and partition of quantities were more frequently used to solve partitive tasks. A small percentage of procedures based on repeated subtraction was observed, suggesting that repeated subtraction cannot be considered as an intuitive procedure associated with division.
\end{abstract}

Key-words: partitive division; quotitive division; arithmetic

$\mathrm{E}$ ste trabalho examina o desempenho de crianças em tarefas de divisão resolvidas por cálculo mental. Dois pontos nos parecem cruciais nesta investigação. $\mathrm{O}$ primeiro diz respeito à relevância do estudo das estratégias orais de solução das operações aritméticas. A segunda refere-se à escolha da divisão como objeto de estudo. Utilizaremos aqui a expressão cálculo mental para designar o cômputo realizado sem a utilização de algoritmos escritos. É o que comumente se denomina "fazer contas de cabeça". Utilizaremos, também, no mesmo sentido, a expressão cálculo oral.

Durante muito tempo, o cálculo mental foi negligenciado pelo currículo escolar, tendo sido, muitas vezes, sinônimo de memorização mecânica de fatos numéricos. No entanto, a descrição de estratégias de cálculo mental utilizadas pelos indivíduos para resolver operações aritméticas presentes em situações de seu cotidiano revela que, longe de ser efetuado apenas pela simples evocação de fatos numéricos, o cálculo oral utiliza-se de diversos invariantes lógico-matemáticos, muitos deles presentes no uso do algoritmo escrito, como, por exemplo, as propriedades associativa, distributiva e comutativa (Nunes, Schliemann, \& Carraher, 1993).

Porque não é implementado por um sistema de regras fixas como acontece com um algoritmo, o sistema oral de cálculo requer a compreensão do usuário acerca dos invariantes lógico-matemáticos em uso, sendo, portanto, o estudo deste sistema de cálculos relevante para a compreensão da construção de conceitos matemáticos. Finalmente, poder-se-ia argumentar que, em vários casos, é mais fácil realizar um determinado cômputo utilizando o cálculo oral do que o algoritmo escrito, principalmente, em situações quotidianas. Isto faz do uso do cálculo oral uma alternativa à utilização do algoritmo escrito em determinadas circunstâncias.

Em relação ao estudo da divisão, há duas décadas investigações vêm sendo realizadas sobre o desenvolvimento das operações multiplicativas (Brown, 1981; Bell, Fischbein, \& Greer, 1984; Bell, Greer, Grimson, \& Magan, 1989; Graeber \& Tirosh, 1988; Graeber, Tirosh, \& Glover, 1989; Greer, 1988, 1989; Vinner \& Linchevski, 1988). Tais pesquisas têm analisa- 
do principalmente diferenças no desempenho de alunos das séries mais avançadas e de professores primários em tarefas de divisão, tendo como fatores a estrutura do problema e o tamanho dos números empregados. A metodologia comumente usada nestes estudos consiste: (a) na tarefa clássica de resolução de problema; (b) na escolha da operação que produz a resposta correta para a solução de um determinado problema e (c) na escrita de um determinado problema para uma operação apresentada.

Embora desde muito cedo as crianças estejam envolvidas com situações quotidianas nas quais devem repartir uma determinada quantidade com familiares e amigos, pouco ainda se conhece sobre o desenvolvimento do conceito de divisão nas crianças até que estas alcancem escolaridade mais avançada.

No estudo da construção inicial do conceito de divisão pela criança é importante distinguir duas classes de problemas: o da divisão partitiva e o da divisão por quotas (Greer, 1992). Nos problemas de divisão partitiva, dados a quantidade a ser dividida e o número de quotas, pergunta-se à criança pelo tamanho da quota. Inversamente, nos problemas de divisão por quotas, é dado o tamanho da quota e pergunta-se, então, pelo número de quotas existentes. $\mathrm{O}$ aspecto partitivo tem sido apontado por alguns autores como o modelo prototípico da divisão (Fischbein, Deri, Nello, \& Marino, 1985). Isto faria com que este tipo de problema seja não só freqüentemente identificado à operação de divisão como, também, resolvido mais facilmente.

Nas últimas décadas, o crescente interesse no desenvolvimento de conceitos matemáticos por crianças gerou uma série de investigações relacionadas principalmente ao desenvolvimento dos conceitos de número, adição e subtração (Carpenter, Hiebert, \& Moser, 1981; Huges, 1986). Tais estudos sublinharam a relevância das estratégias intuitivas da criança para lidar com tais conceitos matemáticos mesmo antes que estes conceitos lhes fossem formalmente ensinados na escola.

Decorrente desta tradição, alguns estudos aparecem na literatura acerca do conhecimento intuitivo da criança sobre a multiplicação e a divisão. Tais investigações podem ser entendidas a partir de dois paradigmas de pesquisa: o Educacional e o da Psicologia do Desenvolvimento. As investigações realizadas do ponto de vista educacional tiveram como objeto principal o exame dos fatores relacionados à situação didática que melhor favorecesse o aprendizado do conceito de divisão pela criança (Burton, 1992; Murray, Olivier, \& Human, 1992; Selva, 1998). Outros trabalhos, como os de Kouba (1989) e Mulligan (1992), que adotaram uma perspectiva de desenvolvimento, estabeleceram como objeto de investigação a descrição detalhada, de acordo com a idade das crianças, das estratégias informais utilizadas para a solução de problemas de multiplicação e de divisão. Apesar da diversidade de propósitos e metodologia, tais estudos mostraram que as crianças são capazes de resolver problemas de divisão muito antes de serem formalmente ensinadas sobre este conceito na escola.
Murray et al. (1992) observaram uma turma de crianças de 8 anos durante um ano letivo. A estratégia didática usada nesta turma consistia em apresentar problemas realistas de divisão partitiva e por quotas às crianças para que estas os resolvessem de acordo com os conhecimentos e procedimentos que já possuíssem. Procurou-se, neste estudo, adotar um procedimento didático diferente do modelo escolar tradicional de solução de problemas, isto é, do modelo segundo o qual a criança é ensinada a identificar previamente a operação aritmética para a solução do problema, escrever a sentença matemática correspondente e finalmente calcular a resposta correta.

Inicialmente as crianças se mostraram bastante influenciadas pela estrutura semântica do problema. Somente com o decorrer do ano letivo, as crianças passaram progressivamente a desenvolver estratégias mais genéricas para resolver os diversos tipos de problemas de divisão. Tais estratégias foram construídas pelas crianças a partir dos procedimentos relacionados à divisão por quotas. $\mathrm{O}$ uso da correspondência termo-a-termo, procedimento característico da representação direta dos problemas partitivos, foi, então, progressivamente abandonado.

A solução dos problemas variou também de acordo com a habilidade matemática dos alunos. Estudantes mais habilidosos em matemática desenvolveram, após alguns meses de trabalho, uma estratégia geral para trabalhar com os problemas de divisão envolvendo a multiplicação, adição e/ou subtração. Os alunos com menor habilidade em matemática levaram mais tempo para abandonar as estratégias baseadas na representação direta dos problemas, apresentando maior dificuldade no desenvolvimento de estratégias mais gerais para a solução dos problemas de divisão baseadas na multiplicação.

Dada a importância conferida pelos professores ao uso de materiais concretos no ensino da matemática elementar, Burton (1992) examinou a influência do uso destes recursos na solução de problemas de divisão partitiva e por quotas por crianças cursando a $2^{\underline{a}}$ série do ensino elementar. Para metade dos problemas apresentados, as crianças poderiam escolher o tipo de material com o qual gostariam de trabalhar: objetos mencionados no problema ou materiais sem nenhuma relação com a história contada no problema (material de contagem). Para a outra metade dos problemas apenas o material de contagem foi deixado como auxiliar.

Os resultados mostraram que o tipo de material não foi importante para o sucesso na resolução dos problemas. O tipo de problema também não influenciou o desempenho das crianças. Não houve diferença significativa entre o sucesso na solução dos problemas de divisão partitiva e por quotas, ou mesmo em relação às estratégias empregadas. A maioria das crianças resolveu ambos os tipos de problema de divisão usando como estratégia básica a formação de conjuntos equivalentes com os objetos disponíveis segundo a quantidade especificada no problema.

Ainda com o objetivo de examinar a influência do uso de material concreto para a solução de problemas de divisão, Selva (1998) comparou o desempenho de crianças com esco- 
laridade entre a Classe de Alfabetização e a $2^{\underline{a}}$ série do Ensino Fundamental em problemas de divisão partitiva e por quotas. As crianças foram divididas em três grupos. O primeiro recebeu material concreto para auxiliá-lo na solução dos problemas; o segundo lápis e papel, e o terceiro nenhum material.

Observou-se que o número de acertos aumentou progressivamente com a escolaridade das crianças, não havendo, no entanto, diferenças no desempenho das crianças nos dois tipos de problemas de divisão apresentados. Também não houve diferença apreciável no desempenho dos grupos que trabalharam com o material concreto e com papel e lápis respectivamente. $\mathrm{O}$ grupo que não recebeu qualquer tipo de material teve desempenho significativamente inferior ao dos dois outros grupos.

Selva (1998) descreveu cinco tipos de estratégias usadas pelas crianças que obtiveram sucesso na solução dos problemas. São elas: (a) representação direta do problema usando a distribuição das quantidades; (b) representação direta com formação de grupos; (c) ensaio e erro; (d) repetição aditiva e (e) emprego de fatos conhecidos.

A freqüência relativa no emprego da representação direta para a solução dos problemas foi maior nos grupos em que a criança podia usar o material concreto como auxiliar. $\mathrm{O}$ uso de estratégias baseadas na repetição aditiva predominou no grupo que não recebeu qualquer material como auxiliar. Selva (1998) argumenta que a presença de materiais concretos que representem numericamente as quantidades envolvidas no problema pode inibir o emprego de estratégias mentais mais sofisticadas pelas crianças.

Voltando nossa atenção agora aos estudos que adotam uma perspectiva baseada na Psicologia do Desenvolvimento, Kouba (1989) realizou um estudo transversal com crianças de 6 a 8 anos, com escolaridade entre a $1^{\underline{a}}$ e $3^{\underline{a}}$ séries, com o propósito de descrever as estratégias intuitivas utilizadas na resolução de problemas de multiplicação e divisão.

A classificação das estratégias realizadas por Kouba (1989) foi baseada no grau de abstração nelas envolvido, sendo descritas cinco categorias: (a) representação direta do problema através de materiais concretos; (b) dupla contagem, isto é, contagem paralela envolvendo o número de elementos em cada conjunto e o número de conjuntos formados; (c) contagem a partir de um fator (contar de cinco em cinco, por exemplo); (d) adições e subtrações repetidas; (e) conhecimento prévio de fatos numéricos relacionados à divisão e à multiplicação.

A estratégia de modelagem direta do problema foi usada pela maioria das crianças nas idades de 6 e 7 anos. Fatos numéricos relacionados à multiplicação e à divisão foram empregados, predominantemente, pelas crianças mais velhas. As estratégias aditivas foram pouco utilizadas pelas crianças.

Os problemas de divisão por quotas não foram considerados pelas crianças mais difíceis que os de divisão partitiva, nem os problemas de divisão foram tomados como mais difíceis que os de multiplicação.

Mulligan (1992), em estudo longitudinal, acompanhou o desenvolvimento das estratégias de solução dos problemas multiplicativos por crianças dos 7 aos 8 anos de idade, descrevendo as seguintes categorias: (a) grupamento: formar conjuntos equivalentes segundo as quantidades especificadas no problema; (b) grupamento por tentativa e erro: a quantidade para a formação dos grupos é estimada, uma vez que esta estimativa não esteja correta, o número de elementos nos conjuntos é aumentado ou diminuído; (c) contagem um a um das quantidades envolvidas no problema; (d) contagem envolvendo um dado fator; (e) dupla contagem; (f) adição repetida; (g) subtração repetida; (h) metades: dividir, por visualização ou concretamente, a quantidade em dois conjuntos equivalentes; (i) correspondência termo-a-termo; (j) correspondência um-para-muitos; (l) uso de conhecimentos prévios de adição; (m) uso de conhecimentos prévios de multiplicação; (n) uso de conhecimentos prévios de divisão; e (o) fatos derivados, ou seja, usar um fato numérico conhecido para encontrar outro fato numérico.

À semelhança de Kouba (1989), pouca diferença foi encontrada por Mulligan (1992) em termos das estratégias usadas para resolver os problemas de multiplicação e divisão. Os procedimentos usados pelas crianças mais novas eram largamente baseados no grupamento e na contagem. $\mathrm{O}$ uso de fatos conhecidos relacionados à adição e à multiplicação aumentou somente por volta de oito anos.

Algumas conclusões gerais podem ser delineadas a partir das evidências empíricas presentes nos estudos acima citados. Inicialmente vê-se relatado, nos diferentes trabalhos, o uso pelas crianças de diversas estratégias intuitivas para a solução de problemas de divisão, mesmo quando este não era o objetivo propriamente dito da investigação. Muitas vezes, tais estratégias aparecem nos vários estudos com nomenclaturas diferentes.

De maneira geral, vê-se uma distinção clara no modo de solução dos problemas de divisão de acordo com a idade. As crianças mais novas tendem a usar procedimentos que modelem a situação problema, enquanto as crianças mais velhas usam mais freqüentemente fatos aritméticos para resolvê-los.

Conforme argumenta Selva (1998), mesmo levando-se em conta a maior influência que fatores de natureza contextual têm no raciocínio das crianças mais novas, a disponibilidade de uso do material concreto pode ter feito com que, nestes estudos, mais crianças usassem procedimentos de modelagem direta do problema quando poderiam ser capazes de usar estratégias mais elaboradas na resolução dos mesmos problemas.

Por outro lado, os estudos apresentados não consideram o impacto do aprendizado formal de outros conceitos aritméticos (como os de adição e subtração) no desenvolvimento das estratégias de solução de problemas de divisão pela criança, tomando como referência, apenas, a idade cronológica das crianças ao relatar o progresso na utilização de estratégias mais sofisticadas de cálculo.

Neste sentido, o presente trabalho examina o desempenho de crianças de 6 a 9 anos com diferentes níveis de escolaridade em aritmética na solução oral de problemas de divisão partitiva e por quotas, descrevendo as estratégias de resolução empregadas por estas crianças. Os estudos relata- 
dos a seguir fazem parte de investigação mais ampla acerca da compreensão do conceito de divisão pela criança descrita por Correa (1995) ${ }^{2}$.

\section{Estudo I: divisão partitiva \\ Método}

\section{Participantes}

Tomaram parte neste estudo: (1) 20 crianças de 6 anos, (10 meninos e 10 meninas; média de idade: 6 anos e 6 meses); (2) 21 crianças de 7 anos (9 meninos e 12 meninas; idade média: 7 anos e 5 meses); (3) 21 crianças de 8 anos (13 meninos e 8 meninas; idade média: 8 anos e 6 meses) e (4) 20 crianças de 9 anos (10 meninos e 10 meninas; idade média: 9 anos 6 meses). As crianças freqüentavam uma escola pública em um bairro de baixo status socioeconômico na cidade de Oxford, Reino Unido. Os grupos estavam distribuídos segundo quatro momentos de sua instrução formal em aritmética: (a) aos 6 anos, antes de qualquer aprendizado formal nas diversas operações aritméticas, porém recebendo instrução em contagem, escrita de números e conhecimentos elementares de fatos numéricos; (b) aos 7 anos, recebendo instrução em adição e subtração; (c) aos 8 anos, ao iniciarem o aprendizado das operações multiplicativas e, finalmente, (d) aos 9 anos, após o recebimento de tal instrução.

\section{Tarefas}

As crianças foram apresentadas a uma situação na qual certa quantidade de blocos (representando comida) deveria ser repartida entre um determinado número de ursinhos de pelúcia. Era pedido, então, às crianças que especificassem o número de blocos que cada ursinho iria ganhar. Os ursinhos de pelúcia, protagonistas da situação problema, eram, à época, personagens extremamente populares entre as crianças inglesas. Foram usados, neste estudo, quatro tamanhos de dividendo (4, 8, 12 e 24) e dois divisores (2 e 4).

\section{Procedimentos}

As crianças foram entrevistadas individualmente. Os ursinhos eram colocados na mesa, sendo a criança convidada a contá-los junto com a pesquisadora. Em seguida, os blocos eram apresentados às crianças para serem divididos igualmente pelos ursinhos. A criança era novamente convidada a contar o número de blocos junto com a pesquisadora. A experimentadora distribuía os blocos entre os ursinhos, colocando-os numa caixa amarrada às costas dos ursinhos. Perguntava-se, então, às crianças quantos blocos cada ursinho teria em sua caixa. Após cada resposta, não importando a sua correção, era pedido que a criança explicasse como a obteve. A ordem de apresentação das tarefas foi randomizada para cada criança.

\section{Resultados}

Os resultados foram analisados de duas maneiras: através do número de respostas corretas dadas às tarefas e pelo tipo de explicação dada pela criança para encontrar o resultado.

\section{O sucesso na tarefa}

Uma vez que os resultados foram codificados em termos binários (acerto e erro), foi utilizada regressão logística para estabelecer a importância de três fatores e sua interação para a compreensão do desempenho das crianças. São estes fatores: (a) idade/escolaridade, uma vez que estas variáveis se confundem neste estudo; (b) dividendo; e (c) divisor.

Os resultados mostraram que os escores obtidos pelas crianças estavam significativamente relacionados à idade/ escolaridade $\left(\chi^{2}=146,701 ; g l=3 ; p<0,001\right)$, ao tamanho do dividendo $\left(\chi^{2}=216,416 ; g l=3 ; p<0,001\right)$ e do divisor $\left(\chi^{2}=\right.$ $12,335 ; g l=1 ; p<0,001)$. Nenhuma interação foi significativa.

Através da inspeção da proporção média de respostas corretas pelos três fatores, pode-se observar o progressivo aumento do número de respostas corretas às tarefas de acordo com a idade/escolaridade (Tabela 1). As crianças obtiveram melhores escores nas tarefas em que se usou um número menor para o dividendo e para o divisor.

\section{As explicações dadas pelas crianças}

A análise de conteúdo dos protocolos das entrevistas revelou dois tipos principais de explicação dados pelas crianças. O primeiro tipo refere-se às explicações que contêm, na verdade, justificativas sobre a plausibilidade dos resultados alcançados pelas crianças. Este tipo de explicação ocorreu

Tabela 1

Proporção média de acertos nas diversas tarefas de divisão por idade

\begin{tabular}{lcccc}
\hline & \multicolumn{4}{c}{ Idade (anos) } \\
\cline { 2 - 5 } $\begin{array}{l}\text { Divisão } \\
\text { Partitiva }\end{array}$ & 6 & 7 & 8 & 9 \\
\hline $4 \div 2$ & 0,75 & 0,95 & 1,00 & 1,00 \\
$4 \div 4$ & 0,70 & 0,90 & 1,00 & 1,00 \\
$8 \div 2$ & 0,40 & 0,71 & 0,95 & 0,95 \\
$8 \div 4$ & 0,30 & 0,66 & 0,80 & 1,00 \\
$12 \div 2$ & 0,20 & 0,57 & 0,80 & 0,90 \\
$12 \div 4$ & 0,20 & 0,42 & 0,57 & 0,85 \\
$24 \div 2$ & 0,05 & 0,38 & 0,57 & 0,90 \\
$24 \div 4$ & 0,10 & 0,19 & 0,52 & 0,80 \\
\hline Divisão por Quotas & & & & \\
\hline $4 \div 2$ & 0,55 & 0,90 & 0,95 & 1,00 \\
$4 \div 4$ & 0,45 & 0,75 & 0,85 & 0,95 \\
$8 \div 2$ & 0,30 & 0,60 & 0,90 & 0,95 \\
$8 \div 4$ & 0,40 & 0,75 & 0,80 & 1,00 \\
$12 \div 2$ & 0,10 & 0,35 & 0,75 & 0,85 \\
$12 \div 4$ & 0,20 & 0,50 & 0,75 & 0,90 \\
$24 \div 2$ & 0,10 & 0,20 & 0,50 & 0,80 \\
$24 \div 4$ & 0,05 & 0,15 & 0,50 & 0,75 \\
\hline & & & &
\end{tabular}


apenas na tarefa em que havia quatro blocos a serem distribuídos para quatro ursinhos, ou seja, quando dividendo e divisor eram iguais.

Por sua vez, o segundo tipo de explicação refletia o tipo de estratégia de cálculo mental utilizado pela criança para alcançar uma determinada resposta. Tal tipo de explicação seguiu-se às outras tarefas de divisão apresentadas.

Na situação em que se procurava saber quanto cada um dos quatro ursinhos trazia na caixa, tendo sido distribuídos quatro blocos entre eles, a resposta da criança podia ser imediata, uma vez que colocasse dividendo e divisor em correspondência termo-a-termo. Para as outras tarefas, no entanto, a criança deveria realizar algum tipo de cálculo mental para chegar à sua resposta, sendo esta a razão pela qual, somente as explicações dadas a estas tarefas são descritas e analisadas neste trabalho.

Dois juízes independentes classificaram as respostas das crianças nas categorias descritas. A percentagem de acordo entre os juízes foi de $96 \%$. Os casos discordantes foram apresentados a um terceiro juiz, cuja decisão foi tomada como final.

Foram descritas 11 categorias. Procurou-se seguir a classificação já feita em estudos anteriores (Kouba, 1989; Mulligan, 1992), mas nem sempre isto foi possível. Em alguns casos estendeu-se o escopo de uma determinada categoria (Categoria X). Em outros casos, nova categoria teve que ser formulada (Categoria XI). As categorias foram as seguintes:

I. Respostas sem explicação, sendo incluídas também nesta categoria respostas como "não sei".

II. Respostas com explicação arbitrária ou idiossincrática. Incluiu-se também nesta categoria respostas que mencionavam alguma competência ou habilidade da criança para resolver o problema. Exemplos: “eu pensei muito”, “eu sou bom em matemática”, “eu sei como fazer contas”.

III. Distribuição um a um: as crianças tentavam modelar a situação através dos dedos, correspondendo um dedo para cada ursinho por vez.

IV. Recontagem das quantidades já apresentadas no problema: geralmente a criança contava de um em um até atingir o valor do dividendo. Exemplo: [tarefa 12 , 2]. A criança ia apontando alternadamente para os ursinhos, contando: “um, dois, três... doze”.

V. Contagem a partir de um dado fator. Exemplo: [tarefa 8 , 4] - "Eu contei: dois, quatro, seis e oito. Então é dois pra cada um”. Foram classificadas nesta categoria somente as respostas em que a contagem foi realizada sem interrupções, ou seja, sem que houvesse a necessidade de se computar subtotais para cada passo.

VI. Dupla contagem: a criança realizava a distribuição em cada rodada contando até alcançar o valor do dividendo. Simultaneamente, contava quantas rodadas haviam sido necessárias para completar a tarefa. Exemplo: tarefa [12, 4] "Três. Eu contei assim: um, dois, três, quatro (levanta um dedo); cinco, seis, sete, oito (levanta outro dedo); nove, dez, onze, doze (levanta outro dedo)”.

VII. Adição repetida: a criança adiciona uma determinada quantidade repetidas vezes até o valor do dividendo ser atin- gido. A criança geralmente precisa calcular os subtotais em cada etapa ou faz menção explícita a termos que se referem ao uso da adição como: e, mais, juntei, tudo junto, etc. Exemplos: [Tarefa 12 , 4] - "Três. Eu fiz três mais três, mais três, mais três. Eu comecei com dois, mas não era. Eu fui então pra três”; [Tarefa 24 , 2] - "Doze. Eu comecei com 6, e eu juntei e vi que não dava. Eu juntei 7 , também não deu. Juntei 8 , juntei 9 , juntei 10, juntei 11 e juntei 12 e fez 24".

VIII. Subtração repetida: a criança subtrai um valor repetidas vezes do dividendo até esgotá-lo. Exemplo: [Tarefa 4, 2] - "Dois. Porque quatro menos dois é dois. Então você põe dois ali, e dois ali”.

IX. Metades. A criança divide sucessivamente em metades o valor correspondente ao dividendo. Exemplo: [Tarefa 8 , 4] - "Dois. Eu parti em quatro e depois em dois. Ficou quatro dois”. É importante notar que a idéia de metade para as crianças mais jovens está fortemente relacionada à idéia de somar o mesmo número duas vezes, como no exemplo a seguir: [tarefa 24 , 2] - “Se 12 e 12 é vinte e quatro e 24 cortado em metades é 12, então eu sei a resposta direto”.

$\mathrm{X}$. Conhecimento de fatos multiplicativos: a criança utiliza conhecimentos aprendidos sobre a divisão ou multiplicação. Exemplo [Tarefa 12 , 2] - "Seis. Porque duas vezes seis é doze”.

XI. Partição e partição associada com produtos: a criança decompõe o dividendo em uma soma de números inteiros de modo a facilitar o cômputo ${ }^{3}$. Exemplo: [Tarefa 12 , 2] “Seis. Cinco ali, cinco lá. Um ali e um lá”.

Tentando identificar os teoremas-em-ação ${ }^{4}$ (Vergnaud, 1982) que subjazem à resposta desta criança, notamos que, inicialmente o 12 é decomposto numa soma $(10+2)$. Depois é gerada outra partição para cada uma das parcelas: $(5+5)+(1+$ 1). Finalmente, é aplicada a propriedade associativa, chegando à partição final $(6+6)$. Podemos resumir o que foi dito acima com a seguinte notação: $[12=10+2=(5+5)+(1+1)=6+6]$.

No caso da partição estar associada a fatos multiplicativos, utiliza-se a propriedade distributiva, ao invés da associativa, e a fatoração. Exemplo: [Tarefa 24, 2] - "Doze. Fiz duas vezes 10 e dois uns. Neste caso a criança realiza a partição de 24 na soma $(20+4)$. Em seguida, transforma cada parcela em um produto para proceder à fatoração conforme demonstrado na notação a seguir: $[24=(2 \times 10)+(2 \times 2)=2 \times(10+2)]$.

Diferenças nos tipos de estratégias usadas pelas crianças foram inicialmente analisadas em função: (a) da idade/ escolaridade das crianças; (b) do tamanho do dividendo; e (c) do valor do divisor. Finalmente, foi analisada a relação entre o tipo de explicação dada pela criança e o seu índice de acertos.

Observa-se que a freqüência de respostas sem explicação ou acompanhadas de explicações arbitrárias decresce consideravelmente com o aumento da idade/escolaridade (Tabela 2). Por contraste, o uso de divisão das quantidades em metades aumenta com a idade/escolaridade, apesar desta não ter sido uma das estratégias usadas com mais freqüência pelas crianças. A subtração repetida raramente foi usada pelas crianças.

Aos 8 anos ocorre a maior freqüência de uso de procedimentos baseados na adição repetida, enquanto que aos 9 
anos aparece um maior índice de procedimentos baseados no uso de fatos multiplicativos. Em ambos os casos, a maior freqüência de uso da estratégia ocorreu um ano depois de iniciada a instrução formal das crianças na operação aritmética envolvida no procedimento empregado. A instrução formal das crianças em adição e subtração se fez aos 7 anos enquanto em multiplicação e divisão, aos 8 anos.

Em relação ao dividendo (Tabela 3), o número de respostas sem explicação ou acompanhadas de explicação arbitrária ou idiossincrática tendeu a aumentar de acordo com o aumento do tamanho do dividendo. No entanto, o inverso ocorre para os procedimentos de adição repetida e uso de metades. Apesar da estratégia IX ocorrer em menos de 10\% dos casos, há mais chance dela ser empregada com um dividendo maior. Não houve diferença apreciável na distribuição das estratégias de cálculo mental em função dos divisores empregados.
A Tabela 4 mostra a distribuição das explicações dadas pelas crianças em função das respostas corretas e incorretas. Em média 87\% das respostas incorretas e apenas 18\% das respostas corretas estavam relacionadas à falta de explicação ou à explicação arbitrária ou idiossincrática.

\section{Discussão}

No que se refere ao desenvolvimento na criança das competências de cálculo mental para lidar com problemas de divisão partitiva, os resultados mostram que o desempenho das crianças foi influenciado pelo tamanho dos números apresentados. O aumento nos valores dos números empregados para o dividendo e o divisor causou considerável dificuldade, principalmente para as crianças mais jovens.

O desempenho das crianças, no entanto, melhorou progressivamente com o aumento da idade/escolaridade. Uma

Tabela 2

Distribuição das estratégias usadas nas tarefas de divisão partitiva em função da idade (em \%)

\begin{tabular}{cccccccccccc}
\hline & \multicolumn{10}{c}{ Estratégias } \\
\cline { 2 - 11 } Idade & I & II & III & IV & V & VI & VII & VIII & IX & X & XI \\
\hline 6 & 22,2 & 55,0 & & 5,0 & 1,4 & 0 & 13,6 & 0,7 & 1,4 & 0 & 0,7 \\
7 & 31,3 & 26,5 & 2,0 & 1,4 & 4,8 & 3,4 & 15,0 & 0,7 & 6,8 & 5,4 & 2,7 \\
8 & 20,4 & 6,1 & 2,0 & 2,7 & 2,0 & 4,8 & 32,0 & 0,7 & 13,6 & 4,1 & 11,6 \\
9 & 5,7 & 4,3 & 0,7 & 0 & 4,3 & 2,8 & 10,0 & 0 & 25,0 & 43,6 & 3,6 \\
\hline
\end{tabular}

Tabela 3

Distribuição das estratégias usadas nas tarefas de divisão partitiva em função do dividendo (em \%)

\begin{tabular}{cccccccccccc}
\hline & \multicolumn{11}{c}{ Estratégias } \\
\cline { 2 - 11 } Dividendo & I & II & III & IV & V & VI & VII & VIII & IX & X & XI \\
\hline 4 & 13,4 & 12,4 & 0 & 0 & 1,4 & 2,5 & 34,7 & 2,5 & 20,8 & 9,8 & 2,5 \\
8 & 14,0 & 19,5 & 3,7 & 1,8 & 4,9 & 1,8 & 22,6 & 0 & 14,6 & 15,3 & 1,8 \\
12 & 23,8 & 26,8 & 0,6 & 1,2 & 4,3 & 3,7 & 11,6 & 0,6 & 8,5 & 14,6 & 4,3 \\
24 & 25,6 & 27,4 & 0 & 4,2 & 1,2 & 3,0 & 11,0 & 0 & 7,3 & 11,0 & 9,3 \\
\hline
\end{tabular}

Tabela 4

Distribuição das estratégias usadas nas tarefas de divisão partitiva em função dos acertos e erros (em \%)

\begin{tabular}{crcccccccccc}
\hline & \multicolumn{110}{c}{ Estratégias } \\
\cline { 2 - 12 } & I & II & III & IV & V & VI & VII & VIII & IX & X & XI \\
\hline Acerto & 7,8 & 10,0 & 1,4 & 0 & 5,0 & 3,6 & 25,0 & 0,8 & 18,7 & 20,8 & 6,9 \\
Erro & 40,7 & 44,4 & 0,9 & 5,6 & 0 & 1,4 & 5,6 & 0 & 0 & 0,5 & 0,9 \\
\hline
\end{tabular}


vez que estas variáveis se confundem em nosso estudo, podemos apenas especular a importância do papel da escolaridade no desenvolvimento e uso espontâneo de conhecimentos aritméticos na solução de situações-problema envolvendo a divisão partitiva. É interessante notar que, como sugerem os resultados obtidos para os grupos de 8 e 9 anos, um aumento apreciável na freqüência de uso espontâneo de procedimentos quer aditivos ou multiplicativos ocorreu no ano que se seguiu à instrução recebida em determinada operação aritmética.

A estratégia de subtração repetida foi raramente empregada pelas crianças nos diversos níveis de escolaridade. Este resultado, similar aos obtidos por Kouba (1989), Mulligan (1992) e Selva (1998), sugere que a subtração repetida não pode ser considerada como um procedimento de cálculo intuitivo para a divisão partitiva.

É importante observar, no entanto, que as situações relacionadas ao modelo partitivo não são as únicas experiências que a criança tem, ao longo de seu desenvolvimento, em relação ao conceito de divisão. Situações-problema envolvendo a divisão por quotas são passíveis de serem encontradas não só no quotidiano das crianças como também ao longo de sua experiência escolar. Uma vez que não é ainda claro na literatura o papel desempenhado pelos dois modelos de divisão no desenvolvimento do conceito de divisão pela criança, nem concordância há sobre a dificuldade relativa de cada um destes modelos, o estudo a seguir examina o desempenho de crianças de 6 a 9 anos na resolução de tarefas de divisão por quotas, comparando, ainda, tais resultados àqueles obtidos no Estudo I para a divisão partitiva.

\section{Estudo II: divisão por quotas Método}

\section{Participantes}

Tomaram parte neste estudo: (1) 20 crianças de 6 anos, (12 meninos e 8 meninas; média de idade: 6 anos e 5 meses); (2) 20 crianças de 7 anos (10 meninos e 10 meninas; idade média: 7 anos e 4 meses); (3) 20 crianças de 8 anos (9 meninos e 11 meninas; idade média: 8 anos e 4 meses) e (4) 20 crianças de 9 anos (10 meninos e 10 meninas; idade média: 9 anos e 6 meses). As crianças freqüentavam uma escola pública em um bairro de baixo status socioeconômico na cidade de Oxford, Reino Unido. O grupo estava distribuído segundo quatro momentos de sua instrução formal em aritmética: (a) aos 6 anos, antes de qualquer aprendizado formal nas diversas operações aritméticas, porém recebendo instrução em contagem, escrita de números e conhecimentos elementares de fatos numéricos; (b) aos 7 anos, recebendo instrução em adição e subtração; (c) aos 8 anos, ao iniciarem o aprendizado das operações multiplicativas e, finalmente, (d) aos 9 anos, após o recebimento de tal instrução.

\section{Tarefas}

As crianças foram apresentadas a uma situação na qual certa quantidade de blocos (representando comida) deveria ser distribuída para os ursinhos durante um piquenique. Para cada ursinho seria feito um pratinho, como mostrado num cartão, com uma determinada quantidade dos blocos apresentados, e assim sucessivamente até terminar o conjunto dos blocos. Perguntava-se, então, à criança quantos ursinhos poderíamos chamar para o piquenique fazendo tal distribuição. Foram utilizados, para este estudo, quatro tamanhos de dividendo (4, 8, 12 e 24) e dois divisores (2 e 4).

\section{Procedimentos}

As crianças foram entrevistadas individualmente. Uma série de ursinhos foi colocada na mesa distante da criança de modo que não fosse permitida sua manipulação. A seguir mostrava-se à criança uma determinada quantidade de blocos a ser distribuída para um piquenique. Os blocos seriam colocados em um pratinho e os ursinhos seriam chamados para comê-los. A criança era convidada a contar o número de blocos junto com a pesquisadora. A experimentadora, então, dispunha os blocos na mesa, tendo o cuidado de colocá-los, não em frente à criança, mas onde a criança pudesse vê-los. Os blocos não eram alinhados, mas empilhados, de modo a não permitir o uso da correspondência visual entre ursinhos e blocos. A pesquisadora explicava que, daquela pilha de blocos, deveriam ser tirados alguns deles, colocados em um pratinho, conforme mostrava o desenho no cartão. Seria, então, chamado um ursinho para receber o prato. Assim seria feito até terminar a pilha de blocos. Perguntou-se, em seguida, à criança quantos ursinhos poderiam ser chamados para o piquenique quando fosse terminada a arrumação dos pratos. Após cada resposta, não importando a sua correção, era pedido que a criança explicasse como a obteve. A ordem de apresentação das tarefas foi randomizada para cada criança.

\section{Resultados}

De maneira similar ao Estudo I, os resultados foram analisados em função do sucesso na tarefa e das estratégias relatadas pela criança para a obtenção da resposta.

\section{O sucesso na tarefa}

A exemplo do Estudo I, foi utilizada regressão logística para examinar o efeito de idade/escolaridade, do dividendo e do divisor no desempenho das crianças.

Os resultados da regressão logística mostraram que os escores obtidos pelas crianças estavam significativamente relacionados à idade/escolaridade $\left(\chi^{2}=163,834 ; g l=3 ; p<\right.$ $0,001)$ e ao tamanho do dividendo $\left(\chi^{2}=166,917 ; g l=3 ; p<\right.$ 0,001). A interação entre dividendo e divisor se mostrou significativa $\left(\chi^{2}=12,673 ; g l=3 ; p<0,01\right)$.

Através da inspeção da proporção média de respostas corretas nos vários fatores, pode-se observar o progressivo aumento do número de respostas corretas às tarefas de acordo com a idade/escolaridade (Tabela 1). As crianças obtiveram também melhores escores nas tarefas em que se usou um número menor para o dividendo.

A interação entre o dividendo e o divisor empregados indica que ter o 2 como divisor é de particular ajuda para os valores extremos do dividendo (i.e., quando o tamanho do 
dividendo é pequeno ou grande). Nas tarefas em que o divisor usado foi 4 , as crianças obtiveram melhores resultados para valores intermediários do dividendo.

Uma possível explicação para este resultado reside no fato de que quando valores intermediários são usados (i.e., casos em que a quantidade não é tão pequena que possibilite à criança lembrar de resultado familiar ou em que as quantidades não sejam tão grandes de modo a dificultar o cálculo mental), o número de passos requerido em tarefas de divisão por quotas é menor quando o divisor empregado é o 4 do que quando usamos o 2 como divisor. Isso pode ser importante quando a criança tenta valer-se de um resultado numérico conhecido para ajudá-la a encontrar a resposta certa.

\section{As explicações dadas pelas crianças}

De maneira similar ao ocorrido no Estudo I, as explicações dadas pela criança à tarefa em que deveriam colocar quatro blocos em cada pratinho tendo apenas um total de quatro blocos compreendiam, na verdade, justificativas através das quais as crianças demonstravam a plausibilidade do resultado alcançado. A resposta a esta tarefa poderia ser imediatamente obtida, sem a realização de qualquer cálculo mental, uma vez que a criança colocasse as quantidades em correspondência. Por este motivo, serão apresentadas, neste trabalho, as análises das explicações referentes às demais tarefas de divisão por quotas. Para estas tarefas, as explicações das crianças deveriam focalizar as estratégias de cálculo utilizadas para a obtenção da resposta.

Dois juizes independentes analisaram as respostas das crianças segundo categorias derivadas da análise de conteúdo dos protocolos. O índice de acordo entre os juízes foi de $97 \%$. Os julgamentos discrepantes foram apresentados a um terceiro juiz, sendo sua decisão tomada como final.

Na descrição das categorias, procurou-se seguir, sempre que possível, a classificação presente na literatura (Kouba, 1989; Mulligan, 1992). No entanto, uma nova categoria foi incluída no estudo (Categoria X).

I. Respostas sem explicação.

II. Respostas com explicação arbitrária ou idiossincrática.

III. Repetição da informação dada.

IV. Distribuição muitos-para-um: a criança distribui os elementos em porções de mais de uma unidade por vez. Exemplo: [Tarefa 4,2 ] - "Dois; porque você tem 4 cubos e você só pode dar comida para este ursinho e este ursinho; dois para um ursinho e dois para o outro ursinho".

V. Dupla contagem. Exemplo: [Tarefa: 24,4$]$ - "Seis. Eu contei em quatros; quatro, oito, doze, dezesseis, vinte e vinte e quatro" (a criança levantava um dedo para cada número contado).

VI. Adição repetida. Exemplo: [Tarefa: 4, 2] - "Dois. Porque dois e dois são quatro e você disse dois para cada ursinho”.

VII. Subtração repetida. Exemplo: [Tarefa 24, 4] - "Seis. Eu comecei com 24. Eu contei 4, e 4; dezesseis. De dezesseis eu tirei outro 4; de 12, eu contei 4. Eu fui descendo. De 8, outro 4 é quatro e eu dei este quatro para outro 4".

VIII. Metades: Exemplo: [Tarefa 12 , 4] - "Três. Eu parti no mesmo número. Na minha cabeça eu pensei que tinha dois pratos. Eu parti em seis. Se eu partir novamente vai ficar três em cada um”.

IX. Conhecimento de fatos multiplicativos. Exemplo: [Tarefa: 8 , 4] - "Dois. Porque duas vezes quatro é oito e oito divido por quatro é dois”.

X. Partição e partição associada com produtos. Exemplo: [Tarefa: 24 , 2] - "Doze. Porque 2 vezes 10 é 20, e partindo 4, faz dois. Juntando os dois juntos, faz doze”. Neste caso, a criança começa com a partição de 24 em $20+4$, para prosseguir como se segue: $[20+4=(2 \times 10)+(4,2)=2(10+2)]$.

Observa-se que a freqüência de respostas sem explicação ou seguidas de explicação arbitrária ou idiossincrática decresce progressivamente com o aumento da idade/escolaridade (Tabela 5). O mesmo acontece com as explicações baseadas na repetição de informações já apresentadas no problema.

Inversamente, ocorre o aumento da freqüência de certas estratégias com a idade/escolaridade. É o caso da dupla contagem, do uso de fatos multiplicativos e da estratégia baseada no uso de metades. A partir dos 8 anos, os problemas tendem a ser resolvidos pelo uso da dupla contagem ou por meio de fatos multiplicativos. Parece que a predominância do uso de estratégia baseada em fatos multiplicativos só ocorre após as crianças terem recebido instrução formal em multiplicação e divisão, o que ocorreu por volta dos 9 anos.

Por outro lado, a freqüência de respostas sem explicação e respostas arbitrárias ou idiossincráticas tende a crescer com o tamanho do dividendo (Tabela 6). Três estratégias foram mais utilizadas com baixos valores para o dividendo: (a) adição repetida; (b) divisão em metades e (c) conhecimento de

Tabela 5

Distribuição das estratégias usadas nas tarefas de divisão por quotas em função da idade (em \%)

\begin{tabular}{ccccccccccc}
\hline & \multicolumn{10}{c}{ Estratégias } \\
\cline { 2 - 11 } Idade & I & II & III & \multicolumn{1}{c}{ IV } & \multicolumn{1}{c}{ V } & VI & VII & VIII & IX & X \\
\hline 6 & 40,7 & 25,0 & 14,3 & 3,5 & 4,3 & 5,7 & 0 & 5,7 & 0,8 & 0 \\
7 & 37,1 & 26,4 & 0,7 & 5,0 & 9,3 & 12,9 & 2,9 & 0 & 5,0 & 0,7 \\
8 & 22,9 & 9,3 & 0 & 0,7 & 21,4 & 6,4 & 0 & 5,0 & 33,6 & 0,7 \\
9 & 18,6 & 0 & 0 & 0 & 23,6 & 5,0 & 0 & 12,1 & 40,7 & 0 \\
\hline
\end{tabular}


fatos multiplicativos. A não ser pelo fato da estratégia de metades ser mais usada nas tarefas cujo divisor é 2, não foi notada nenhuma diferença apreciável na distribuição das estratégias utilizadas pelas crianças em função do divisor.

Três categorias são responsáveis por quase 66\% das respostas corretas. São elas: (a) conhecimento de fatos multiplicativos; (b) dupla contagem e (c) adição repetida.

A exemplo dos resultados obtidos no Estudo I, a Tabela 7 mostra que a maioria das respostas incorretas (78\%) ou está associada com a falta de explicação, ou é seguida de explicação arbitrária, enquanto somente 20\% das respostas corretas foram associadas a essas categorias.

\section{Análise conjunta dos estudos I e II}

Uma vez que o delineamento experimental usado nos dois estudos foi idêntico, uma análise conjunta dos dois estudos foi feita de maneira a examinar se haveria diferenças significativas no desempenho das crianças nas tarefas de divisão partitiva e por quotas. A análise foi feita em função do sucesso na tarefa e das explicações dadas pelas crianças para a obtenção do resultado.

\section{O sucesso nas tarefas de divisão partitiva e por quotas}

As respostas das crianças foram submetidas a uma análise de regressão logística, examinando a importância dos seguintes fatores para a compreensão do desempenho das crianças nas tarefas: (a) tipo de divisão; (b) idade/escolaridade; (c) dividendo e (d) divisor.
Essa análise revelou que os escores das crianças estavam significativamente relacionados à idade/escolaridade $\left(\chi^{2}\right.$ = 309,367; $g l=3 ; p<0,001)$, ao tamanho do dividendo $\left(\chi^{2}=\right.$ 376,968; $g l=3 ; p<0,001)$, ao divisor $\left(\chi^{2}=5,847 ; g l=1 ; p<\right.$ $0,05)$ e ao tipo de divisão $\left(\chi^{2}=4,507 ; g l=1 ; p<0,05\right)$. A interação Dividendo x Divisor x Tipo de Divisão foi significativa $\left(\chi^{2}=13,085 ; g l=3 ; p<0,01\right)$.

A análise do número de respostas corretas para os fatores acima mostra que as crianças com maior escolaridade obtiveram maior sucesso na resolução da tarefa. Observou-se que, geralmente, quanto maiores os valores utilizados para o dividendo e para o divisor, maiores foram também as dificuldades encontradas pelas crianças para a realização dos cálculos.

De modo geral, os problemas de divisão partitiva foram resolvidos mais facilmente pelas crianças. No entanto, o fato da interação entre dividendo, divisor e tipo de divisão ter sido significativa mostra que as observações acima devem ser relativizadas, como também melhor entendidas quando tomamos em consideração determinadas condições da tarefa. Quando foram utilizados valores intermediários para o dividendo (8 e 12) e o número 4 foi o valor empregado para o divisor, as crianças puderam obter escores melhores nas tarefas de divisão por quotas. Nos problemas de divisão por quotas, o incremento no valor do divisor não aumentou necessariamente a dificuldade da tarefa.

\section{Discussão Final}

O desenvolvimento de competências de cálculo mental em crianças para a solução de tarefas de divisão foi examina-

Tabela 6

Distribuição das estratégias usadas nas tarefas de divisão por quotas em função do dividendo (em \%)

\begin{tabular}{ccccccccccc}
\hline & \multicolumn{11}{c}{ Estratégias } \\
\cline { 2 - 11 } Dividendo & I & II & III & IV & \multicolumn{1}{c}{ V } & VI & VII & VIII & IX & X \\
\hline 4 & 22,8 & 10,6 & 5,0 & 5,0 & 3,0 & 18,6 & 0 & 10,6 & 24,4 & 0 \\
8 & 27,3 & 12,0 & 3,1 & 0,5 & 10,6 & 14,8 & 0,5 & 6,4 & 24,8 & 0 \\
12 & 30,6 & 17,5 & 5,0 & 0,8 & 17,5 & 1,3 & 1,3 & 5,5 & 20,5 & 0 \\
24 & 35,7 & 18,8 & 3,2 & 0 & 22,8 & 0,8 & 0,8 & 3,2 & 13,4 & 1,3 \\
\hline
\end{tabular}

Tabela 7

Distribuição das estratégias usadas nas tarefas de divisão por quotas em função dos acertos e erros (em \%)

\begin{tabular}{ccccccccccc}
\hline & \multicolumn{10}{c}{ Estratégias } \\
\cline { 2 - 11 } & I & II & III & IV & V & VI & VII & VIII & IX & X \\
Acerto & 16,4 & 4,8 & 0 & 3,0 & 18,8 & 12,4 & 0,6 & 9,8 & 33,4 & 0,8 \\
Erro & 50,2 & 30,2 & 8,3 & 0,4 & 8,7 & 0,4 & 0,9 & 0 & 0,9 & 0 \\
\hline
\end{tabular}


do tendo como referência o tipo de problema de divisão apresentado às crianças, os valores numéricos presentes nos problemas, bem como os diferentes níveis de instrução em aritmética das crianças nas diversas faixas etárias.

Os resultados obtidos em nossos dois experimentos mostram que o sucesso na resolução das tarefas não está relacionado apenas ao tipo de problema de divisão apresentado, mas é influenciado também pelas quantidades escolhidas para os termos da divisão.

A análise conjunta dos Estudos I e II sugere que a maior facilidade encontrada na solução de tarefas de divisão partitiva depende, em última instância, dos valores numéricos utilizados para o dividendo e para o divisor. Uso de valores intermediários para o dividendo associado ao maior divisor usado para as tarefas apresentadas facilitou o cômputo na solução de tarefas de divisão por quotas.

Uma possível explicação para os resultados encontrados parece estar na combinação de valores para o dividendo e divisor, o que reduziria a quantidade de etapas de cálculo requeridas para a solução de problemas de divisão por quotas. Por exemplo, no caso em que as crianças foram apresentadas à tarefa em que 12 era o dividendo e 4 o divisor, tudo o que a criança precisaria fazer nas tarefas de divisão por quotas seria contar o número de vezes em que a correspondência muitos-para-um entre blocos e ursinhos ocorreria de forma a ter quatro-para-A, quatro-para-B, quatro-para-C e quatropara-D. Por outro lado, no caso da divisão partitiva, a criança teria que imaginar a distribuição de 12 cubos entre 4 ursinhos com base, principalmente, na correspondência termo-a-termo. Mesmo usando, nas tarefas de divisão partitiva, algum tipo de estratégia baseada na estimativa e ajuste para o número de blocos distribuídos de cada vez, esta, ainda sim, não seria uma estratégia mais direta do que aquela usada para o cômputo da tarefa de divisão por quotas.

Não se pode, portanto, a partir de tais resultados, corroborar a idéia de que a divisão partitiva seja o modelo intuitivo de divisão para a criança como sugerido na literatura (Fischbein et al., 1985).

No que se refere às estratégias de cálculo utilizadas, observou-se a descrição de praticamente as mesmas categorias para as tarefas de divisão partitiva e por quotas, conforme a literatura (Kouba, 1989; Mulligan, 1992; Selva, 1998). No entanto, vemos que a importância relativa de determinadas categorias diferiu de um tipo de divisão para o outro, mostrando que a maneira com que as crianças resolveram as tarefas de divisão apresentadas foi sensível à estrutura subjacente à situação-problema.

A análise da tentativa da criança em modelar as relações subjacentes aos problemas de divisão aparece documentada na literatura apenas quando a criança resolve os problemas em presença do material concreto ou quando utiliza os próprios dedos como auxiliares. Murray et al. (1992) e Selva (1998), por exemplo, reportam o emprego da distribuição para a resolução dos problemas de divisão partitiva e a estratégia de formação de pequenos grupos seguida da contagem do número de grupos formados para a divisão por quotas.
A diferença na freqüência dos procedimentos de cálculo observada em nosso estudo segundo o tipo de problema de divisão apresentado sugere a tentativa da criança em modelar as relações envolvidas no problema, desta vez não com o uso do material concreto, mas através de estratégias mentais de cálculo.

Diferentemente do emprego do material concreto para representar diretamente as situações descritas no problema, os procedimentos de cálculo oral utilizados pelas crianças modelam, por sua vez, as relações lógico-matemáticas estabelecidas sobre os fatos numéricos nos problemas apresentados.

Assim sendo, observa-se que os procedimentos de cálculo empregados pelas crianças servem não só para lidarem com diferenças no tamanho dos números presentes em cada um dos problemas, mas, sobretudo, para modelar as operações lógico-matemáticas exercidas sobre estes mesmos valores.

No caso da divisão partitiva, observa-se o aumento na freqüência relativa de estratégias relacionadas à partição dos números, seja em partes iguais (adição repetida e metades), seja em parcelas diferentes (a estratégia denominada neste estudo como partição propriamente dita). Na divisão por quotas, por outro lado, observa-se o uso de estratégias relacionadas às vezes em que uma determinada quantidade pode “estar contida” em outra quantidade, como no caso da dupla contagem.

As crianças usaram também com mais freqüência procedimentos baseados em fatos multiplicativos nos problemas de divisão por quotas. Provavelmente as crianças estariam tirando partido do fato de terem sido ensinadas a gerar as tabuadas de multiplicação (n vezes x dá y), o que facilita encontrar quantas vezes determinada porção poderia ser obtida até chegar à quantidade especificada no dividendo.

Uma porcentagem muito pequena de procedimentos baseados no uso da subtração repetida foi encontrada para ambos os tipos de divisão. Tais resultados, somados aos encontrados por Kouba (1989), Mulligan (1992) e Selva (1998), fornecem evidências empíricas para que a subtração sucessiva não possa ser tomada como um tipo de procedimento intuitivo de cálculo usado pelas crianças para a aquisição inicial do conceito de divisão.

No que se refere ainda à distribuição das estratégias pelos diferentes grupos de crianças entrevistadas, observa-se que o uso de determinadas estratégias de cálculo e a escolaridade parecem estar relacionados. Somente após serem formalmente ensinadas sobre as operações de multiplicação e divisão, as crianças passaram a usar predominantemente uma estratégia multiplicativa para a solução de ambos os tipos de divisão.

De maneira geral, o sucesso na realização da tarefa e a descrição dos procedimentos de solução utilizados estão também relacionados para ambos os tipos de divisão. Menos de $20 \%$ das respostas corretas não foram seguidas de nenhuma explicação, ou foram acompanhadas de uma explicação idiossincrática. 
Importante conclusão pode ser tirada destes últimos resultados. Uma vez que a criança tivesse encontrado um procedimento sistemático de cálculo mental para a solução das tarefas de divisão, seja esta partitiva ou por quotas, ela não só era capaz de descrever claramente tal procedimento como também de encontrar a solução correta para a tarefa proposta. Isso implica considerar que, para a compreensão do conceito de divisão, a habilidade da criança em realizar uma seqüência de procedimentos de cálculo mental para resolver um problema relaciona-se com o entendimento dos princípios operatórios subjacentes à estratégia empregada. A segunda importante implicação diz respeito à relação entre a operação mental realizada e sua representação. A criança parece poder coordenar, nestes casos, suas ações com uma consistente descrição delas.

\section{Referências}

Bell, A., Greer, B., Grimison, L., \& Mangan, C. (1989). Children’s performance on multiplicative word problems: elements of a descriptive theory. Journal for Research in Mathematics Education, 20(5), 434-449.

Bell, A. W., Fischbein, E., \& Greer, B. (1984). Choice of operation in verbal arithmetic problems: the effects of number size, problem structure and context. Educational Studies in Mathematics, 15, 129-147.

Brown, M. (1981). Number operations. In K. M. Hart (Org.), Children's understanding of mathematics: 11 16 (pp. 23-47). Londres: John Murray.

Burton, G. (1992). Young children's choices of manipulatives and strategies for solving whole number division problems. Focus on Learning Problems in Mathematics, 14, 2-57.

Carpenter, T. P., Hiebert, J., \& Moser, J. M. (1981). Problem structure and first grade children's initial solution processes for simple addition and subtraction problems. Journal for Research in Mathematics Education, 12, 27-39.

Correa, J. (1995). Young children's understanding of the division concept. Tese de doutorado não-publicada, Universidade de Oxford, Oxford.

Fischbein, E., Deri, M., Nello, M., \& Marino, M. (1985). The role of implicit models in solving verbal problems in multiplication and division. Journal for Research in Mathematics Education, 16(1), 3-17.
Graeber, A. O., \& Tiroshi, D. (1988). Multiplication and division involving decimals: preservice elementary teachers' performance and beliefs. Journal of Mathematical Behavior, 7, 263-280.

Graeber, A. O., Tiroshi, D., \& Glover, R. (1989). Preservice teachers' misconception in solving verbal problems in multiplication and division. Journal for Research in Mathematics Education, 20(1), 95-102.

Greer, B. (1988). Nonconservation of multiplication and division: analysis of a symptom. Journal of Mathematical Behavior, 7, 281-298.

Greer, B. (1989). Conceptual obstacles to the development of the concepts of multiplication and division. In H. Mandl, E. de Corte, S. N. Bennett, \& H. F. Friedrich (Orgs.), Learning and instruction: European research in an international context (pp. 461-476). Oxford: Pergamon.

Greer, B. (1992). Multiplication and division as models of situations. In D. A Grouws (Org.), Handbook of research on mathematics, teaching and learning (pp. 276-295). Nova York: Macmillan.

Hughes, M. (1986). Children and number: difficulties in learning mathematics. Oxford: Basil Blackwell.

Kouba, V. L. (1989). Children's solution strategies for equivalent set multiplication and division word problems. Journal for Research in Mathematics Education, 20(2), 147-158.

Mulligan, J. (1992). Children's solutions to multiplication and division word problems: a longitudinal study. Mathematics Education Research Journal, 4, 24-41.

Murray, H., Olivier, A., \& Human, P. (1992). The development of young student's division strategies. Proceedings of the Sixteenth International Conference, Psychology of Mathematics Education (pp. 152-159). Durham: Autor.

Nunes, T., Schliemann, A. D., \& Carraher, D. (1993). Street mathematics and school mathematics. Cambridge: Cambridge University Press.

Selva, A. C. V. (1998). Discutindo o uso de materiais concretos na resolução de problemas de divisão. In A. Schliemann \& D. Carraher (Orgs.), A compreensão de conceitos aritméticos: ensino e pesquisa (pp. 95-119). São Paulo: Papirus.

Vergnaud, G. (1982). A classification of cognitive tasks and operations of thought involved in addition and subtraction problems. In T. P. Carpenter \& J. M. Moser (Orgs.), Addition and subtraction: a cognitive perspective (pp. 3959). Hillsdale, New Jersey: Lawrence Erlbaum.

Vinner, S., \& Linchevski, L. (1988). Is there any relation between division and multiplication? Elementary teachers' ideas about division. Proceedings of the Twelfth International Conference, Psychology of Mathematics Education (pp. 625-632). Veszprem: Autor.

\section{Notas}

1 Apoio CNPq

2 Os estudos aqui relatados resultam das investigações apresentadas em tese de doutoramento (Correa, 1995) realizada com apoio do CNPq.

3 Quando a partição envolve a decomposição do número em n partes iguais, esta corresponde à adição repetida.

4 Vergnaud (1982) denomina de teoremas-em-ação aos princípios lógicos, pressupostos e inferências que não são necessariamente expressos ou explicados pela criança, mas que podem ser inferidos através de seu desempenho.

Jane Correa, doutora em Psicologia pela Universidade de Oxford, Reino Unido, é professora adjunta no Instituto de Psicologia da Universidade Federal do Rio de Janeiro. Endereço para correspondência: Programa de Pós-Graduação em Psicologia, Instituto de Psicologia, Universidade Federal do Rio de Janeiro; Av. Pasteur, 250 Fundos, Praia Vermelha; Rio de Janeiro, RJ; CEP 22295-900. 